\title{
Screening of a library of traditional Chinese medicines to identify anti-malarial compounds and extracts
}

\author{
Motohiro Nonaka, Yuho Murata, Ryo Takano, Yongmei Han, Md. Hazzaz Bin Kabir and Kentaro Kato*
}

\begin{abstract}
Background: Malaria is a major infectious disease in the world. In 2015, approximately 212 million people were infected and 429,000 people were killed by this disease. Plasmodium falciparum, which causes falciparum malaria, is becoming resistant to artemisinin (ART) in Southeast Asia; therefore, new anti-malarial drugs are urgently needed. Some excellent anti-malarial drugs, such as quinine or ART, were originally obtained from natural plants. Hence, the authors screened a natural product library comprising traditional Chinese medicines (TCMs) to identify compounds/ extracts with anti-malarial effects.

Methods: The authors performed three assays: a malaria growth inhibition assay (GIA), a cytotoxicity assay, and a malaria stage-specific GIA. The malaria GIA revealed the anti-malarial ability and half-maximal inhibitory concentrations $\left(I C_{50}\right)$ of the natural products, whereas the malaria stage-specific GIA revealed the point in the malaria life cycle where the products exerted their anti-malarial effects. The toxicity of the products to the host cells was evaluated with the cytotoxicity assay.

Results: Four natural compounds (berberine chloride, coptisine chloride, palmatine chloride, and dehydrocorydaline nitrate) showed strong anti-malarial effects $\left(\mathrm{IC}_{50}<50 \mathrm{nM}\right.$ ), and low cytotoxicity (cell viability $\left.>90 \%\right)$ using P. falciparum 3D7 strain. Two natural extracts (Phellodendri cortex and Coptidis rhizoma) also showed strong antiplasmodial effects $\left(\mid \mathrm{C}_{50}<1 \mu \mathrm{g} / \mathrm{ml}\right.$ ), and low cytotoxicity (cell viability $>80 \%$ ). These natural products also demonstrated anti-malarial capability during the trophozoite and schizont stages of the malaria life cycle.

Conclusions: The authors identified four compounds (berberine chloride, coptisine chloride, palmatine chloride, and dehydrocorydaline nitrate) and two extracts (Phellodendri cortex and Coptidis rhizoma) with anti-malarial activity, neither of which had previously been described. The $\mathrm{IC}_{50}$ values of the compounds were comparable to that of chloroquine and better than that of pyrimethamine. These compounds and extracts derived from TCMs thus show promise as potential future anti-malarial drugs.
\end{abstract}

Keywords: Antimalarial drugs, Drug screening, Traditional Chinese medicine, Plasmodium falciparum

\section{Background}

Malaria is major, global infectious disease. In 2015, approximately 212 million people were infected and 429,000 people were killed by this infection, mainly in tropical and subtropical regions [1]. Malaria is caused by various Plasmodium protozoal parasites

\footnotetext{
*Correspondence: kkato@obihiro.ac.jp
}

National Research Center for Protozoan Diseases, Obihiro University

of Agriculture and Veterinary Medicine, Inada-cho, Obihiro, Hokkaido 080-8555, Japan
(Plasmodium falciparum, Plasmodium vivax, Plasmodium malariae, Plasmodium ovale, Plasmodium knowlesi) [2]; falciparum malaria, caused by P. falciparum, is responsible for most of the malaria-related deaths globally and severe cases of malaria [1].

The main drug currently used to treat malaria is artemisinin (ART). Its derivatives are used in artemisininbased combination therapy (ACT) and have helped countless patients all over the world [3]. However, $P$. falciparum is becoming resistant to ART in Southeast

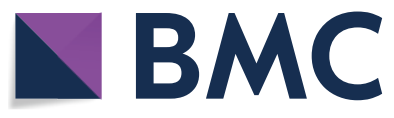

(c) The Author(s) 2018. This article is distributed under the terms of the Creative Commons Attribution 4.0 International License (http://creativecommons.org/licenses/by/4.0/), which permits unrestricted use, distribution, and reproduction in any medium, provided you give appropriate credit to the original author(s) and the source, provide a link to the Creative Commons license, and indicate if changes were made. The Creative Commons Public Domain Dedication waiver (http://creativecommons.org/ publicdomain/zero/1.0/) applies to the data made available in this article, unless otherwise stated. 
Asia. When resistance to chloroquine (CQ) and sulfadoxine (SD)-pyrimethamine (PYR) spread from Southeast Asia and South America to Africa, many African people, especially children, died. To avoid a similar outcome due to ART resistance, the authors must develop new anti-malarial treatment options [4].

Humans have used plants for medicine since ancient times. Indeed, the first anti-malarial drugs, quinine $(\mathrm{QN})$ and quinidine (QND), were found in the bark of the Cinchona tree and have been used for over 350 years as an anti-malarial drug in South America [5]. In China, traditional Chinese medicines (TCMs) have a more than 2000-year history and their appropriate use has been developed over a long time [6]. ART, which has become one of the best known antimalarial drugs, was also discovered from a plant, Artemisia annua. This plant is a famous TCM identified by $\mathrm{Dr}$. Tu who established screening protocols of Chinese traditional herbs and was awarded the Nobel Prize in Physiology or Medicine in 2015 [3].

Clearly, some excellent anti-malarial drugs have been derived from natural plants. Here, the authors were fortunate to obtain a natural product library comprising TCMs. This library contained 96 different compounds and 120 different extracts that had never been evaluated for anti-malarial activity. Many of compounds were refined from the extracts. Hence, the authors screened this natural product library with the aim of identifying compounds and extracts with antimalarial activities. The authors found several hit compounds and extracts that could be candidates for new drug against malaria parasites.

\section{Methods}

\section{Compounds}

The natural drug library was provided by the Institute of Natural Medicine (The University of Toyama, Toyama, Japan). Pyrimethamine (Wako, Osaka, Japan) and artesunate (ATN; Sigma, MO, USA) were also used for the screening, as described below.

\section{Plasmodium falciparum culture in vitro}

The authors used P. falciparum 3D7 and Dd2 strain blood stage parasites for this study. Parasites were grown in $\mathrm{AB}+$ human red blood cells (RBCs) and maintained in culture medium containing RPMI 1640, $25 \mathrm{mM}$ HEPES, $100 \mu \mathrm{M}$ hypoxanthine, $12.5 \mu \mathrm{g} / \mathrm{ml}$ gentamycin, $0.5 \%(\mathrm{w} / \mathrm{v})$ Albumax II, and $62.5 \mu \mathrm{g} / \mathrm{ml}$ $\mathrm{NaHCO}_{3}$. The culture was maintained at $37^{\circ} \mathrm{C}, 5 \% \mathrm{O}_{2}$, and $5 \% \mathrm{CO}_{2}$, with daily medium changes.

\section{Cell culture}

Human embryonic kidney derived (HEK) 293T cells were grown in culture medium containing DMEM, $10 \%$ fetal bovine serum (FBS), L-glutamine, penicillin-streptomycin, and $62.5 \mu \mathrm{g} / \mathrm{ml} \mathrm{NaHCO}_{3}$ at $37{ }^{\circ} \mathrm{C}$, with $5 \% \mathrm{O}_{2}$ and $5 \% \mathrm{CO}_{2}$. The cells were passaged every 2 days at $70-80 \%$ confluency.

\section{Plasmodium falciparum growth inhibition assays (GIAs)}

GIAs were performed as previously described [7]. Briefly, cultures containing mainly ring-stage parasites were synchronized by means of $\mathrm{D}$-sorbitol treatment. After $36 \mathrm{~h}$, cultures containing mostly ring-stage parasites growing up again were again synchronized by use of sorbitol treatment. GIAs were initiated on the next day when the synchronized parasites grew up to the late trophozoite stage. Infected human red blood cells (iRBCs) were mixed with fresh type $\mathrm{AB}+$ uninfected human RBCs (uRBCs) to prepare cultures with $0.3 \%$ parasitaemia and 1\% haematocrit. All compounds were dissolved in DMSO at the concentration of $10 \mathrm{mM}$, and all extracts were dissolved in distilled water at $100 \mathrm{mg} /$ $\mathrm{ml}$. The authors prepared each required concentration of compounds and extracts in serial dilution methods. The cultures were then transferred into 96-well plates at $147 \mu \mathrm{l}$ per well, and $3 \mu \mathrm{l}$ of compounds/extracts were added to each well. All experiments were done in triplicate. The plates were incubated at $37{ }^{\circ} \mathrm{C}$, with $5 \% \mathrm{O}_{2}$ and $5 \% \mathrm{CO}_{2}$. At $48 \mathrm{~h}$ post-incubation, $50 \mu \mathrm{l}$ of complete medium was added to each well. The degree of inhibition was assessed by determining the parasitaemia by using an optical microscope at $96 \mathrm{~h}$ post-incubation when the parasites were mostly at the trophozoite or schizont stages. The authors determined the growth inhibitory rate as follows, growth inhibitory rate $(\%)=[1-\{($ parasitaemia of sample $)-($ parasitaemia of positive control $)\} /\{$ (parasitaemia of negative control) $-($ parasitaemia of positive control $)\}] \times 100$. IC $_{50}$ was determined by analysis of dose-response curve made by GraphPad Prism (GraphPad Software, CA, USA).

\section{Cytotoxicity assays}

Cell Counting Kit-8 (Dojindo, Kumamoto, Japan) was used for the cytotoxicity assays. HEK $293 \mathrm{~T}$ cells were cultured using 96-well microplates in $100 \mu \mathrm{l}$ of medium for $96 \mathrm{~h}$ with various concentrations of hit compounds $\left(10^{1}, 1,10^{-1}, 10^{-2}, 10^{-3} \mu \mathrm{M}\right)$ or extracts $\left(10^{2}\right.$, $\left.10^{1}, 1,10^{-1}, 10^{-2}, 10^{-3} \mu \mathrm{g} / \mathrm{ml}\right)$. After the $96-\mathrm{h}$ incubation, $10 \mu \mathrm{l}$ of Cell Counting Kit solution was added to each well and incubated for $3 \mathrm{~h}$. Then, the absorbance at $450 \mathrm{~nm}$ was measured using a plate reader (Corona Electric, Ibaraki, Japan). The authors determined the 
cell viability as follows, cell viability $(\%)=\{$ (absorbance at $450 \mathrm{~nm}$ of treated group)/(absorbance at $450 \mathrm{~nm}$ of control group) $\} \times 100$. Parasite selectivity is important index to evaluate the practical use of the compounds and extracts for malaria treatment; high numbers have high parasite selectivities. The authors also calculated the parasite selectivity as follows, parasite selectivity $(\%)=\left\{\left(\mathrm{IC}_{50}\right.\right.$ value of host cell $) /\left(\mathrm{IC}_{50}\right.$ value of parasite $)\} \times 100$.

\section{Stage-specific growth inhibition assays}

Stage-specific GIAs were carried out similarly to standard GIAs [8], but stage specificity was initiated immediately after the second synchronization. iRBCs were mixed with fresh type $\mathrm{AB}+\mathrm{uRBCs}$ to prepare cultures with $0.3 \%$ parasitaemia and $1 \%$ haematocrit. Cultures were transferred into a 96-well plate at $147 \mu \mathrm{l}$ per well. Cultures were divided into five groups: ring-stage samples, trophozoite-stage samples, schizont-stage samples, positive control (10 $\mu \mathrm{M}$ PYR), and negative control ( $0.1 \%$ DMSO), and $3 \mu \mathrm{l}$ of compounds/extracts were added simultaneously to each well, first for $0-24 \mathrm{~h}$ into the ringstage wells, next for 24-36 h into the trophozoite wells, and last for 36-48 $\mathrm{h}$ into the schizont wells. After exposure to the compounds/extracts, the RBCs were washed three times with culture medium, and cultured until $48 \mathrm{~h}$ post-incubation. These experiments were performed in triplicate. The cultures were incubated at $37^{\circ} \mathrm{C}$, with $5 \% \mathrm{O}_{2}$ and $5 \% \mathrm{CO}_{2}$. The amount of compounds/extracts was assessed by determining the parasitaemia of Giemsastained samples by using an optical microscope at $48 \mathrm{~h}$ post-incubation when the parasites were mostly in the trophozoite or schizont stages.

\section{Results}

\section{Screening of the natural compound library for anti-Plasmodium effects}

The authors screened 96 natural compounds at a final concentration of $10 \mu \mathrm{M}$ to assess their P. falciparum growth inhibitory effects. Parasitaemia in the wells to which $0.1 \%$ DMSO was added was set as $0 \%$ growth inhibition, whereas that in the wells treated with $10 \mu \mathrm{M}$ PYR, which is enough to inhibit the growth of $P$. falciparum 3D7 strain, was calculated as almost $100 \%$ inhibition. Of these 96 compounds, five (berberine chloride, coptisine chloride, palmatine chloride, dehydrocorydaline nitrate, and timosaponin A-III) inhibited growth by $>99 \%$ (Fig. 1a). This is the first report of anti-malarial activity for dehydrocorydaline nitrate. Since timosaponin A-III had a haemolytic effect, the authors selected the other four compounds (Table 1) for further assessment. Next, the authors determined the half maximal inhibitory concentrations $\left(\mathrm{IC}_{50}\right)$ of the four compounds (Fig. 1b); the $\mathrm{IC}_{50}$ values of all four compounds were $<50 \mathrm{nM}$ (Table 1). Additionally, the authors assessed anti-malarial effect of the four compounds using $P$. falciparum Dd2 strain. $10 \mu \mathrm{M}$ ATN, which is enough to inhibit the growth of $P$. falciparum $\mathrm{Dd} 2$ strain, was used as the positive control. The four compounds inhibited Dd2 strain parasite growth similarly (Fig. 2), and $\mathrm{IC}_{50}$ values were $<200 \mathrm{nM}$; higher than 3D7 strain (Table 2). Previously reported $\mathrm{IC}_{50}$ values for CQ and PYR were $27.1 \mathrm{nM}$ and $733.26 \mathrm{nM}$, respectively $[9,10]$, indicating that the $\mathrm{IC}_{50}$ values of the authors' test compounds were comparable to or better than those of CQ and PYR.

Berberine chloride, coptisine chloride, and palmatine chloride are isoquinoline alkaloids, some of which have previously been shown to have antiplasmodial activity [11]. The authors' data indicate that a dehydrocorydaline nitrate, which has a similar structure to that of an alkaloid, can be added to the list of isoquinoline alkaloid-like compounds with inhibitory activity against plasmodial parasites.

\section{Screening of the natural extract library for anti-Plasmodium falciparum effects}

The authors screened 120 natural extracts at a final concentration of $100 \mu \mathrm{g} / \mathrm{ml}$ for $P$. falciparum 3D7 strain growth inhibitory effects. Parasitaemia in the wells to which only sterilized distilled water was added was set as $0 \%$ growth inhibition, whereas that in the wells treated with $10 \mu \mathrm{M}$ PYR was calculated as $100 \%$ inhibition. Of the 120 extracts screened, 19 inhibited growth by $>90 \%$ (Fig. 3a). The authors chose the nine extracts with the greatest inhibitory effects from these 19 and determined their $\mathrm{IC}_{50}$ values. Three of the nine (Phellodendri cortex, Coptidis rhizoma, and Caryophylli flos) showed strong anti-malarial effects at $20 \mu \mathrm{g} / \mathrm{ml}$ (Fig. 3b), and the $\mathrm{IC}_{50}$ values of two of them (Phellodendri cortex and Coptidis rhizoma) were $<1 \mu \mathrm{g} / \mathrm{ml}$ (Table 3). This is the first-time anti-malarial activity for these three extracts has been reported. Also, the authors determined the anti-malarial effect of the two extracts using $P$. falciparum Dd2 strain. Positive control was $10 \mu \mathrm{M}$ ATN. Dd2 strain parasite growth was inhibited by the extracts similarly (Fig. 4), and $\mathrm{IC}_{50}$ values were higher than those of $3 \mathrm{D} 7$ strain (Table 4).

\section{Cell viability}

The effects of the four compounds and two extracts on the viability of HEK 293T cells was assessed. Absorbance at $450 \mathrm{~nm}$ of wells containing $0.1 \%$ DMSO or sterilized distilled water was set as $100 \%$ cell viability. Cell viability in the presence of any of the four compounds was $>90 \%$ and in the presence of either extract was $>80 \%$ (Fig. $5 \mathrm{a}$, 


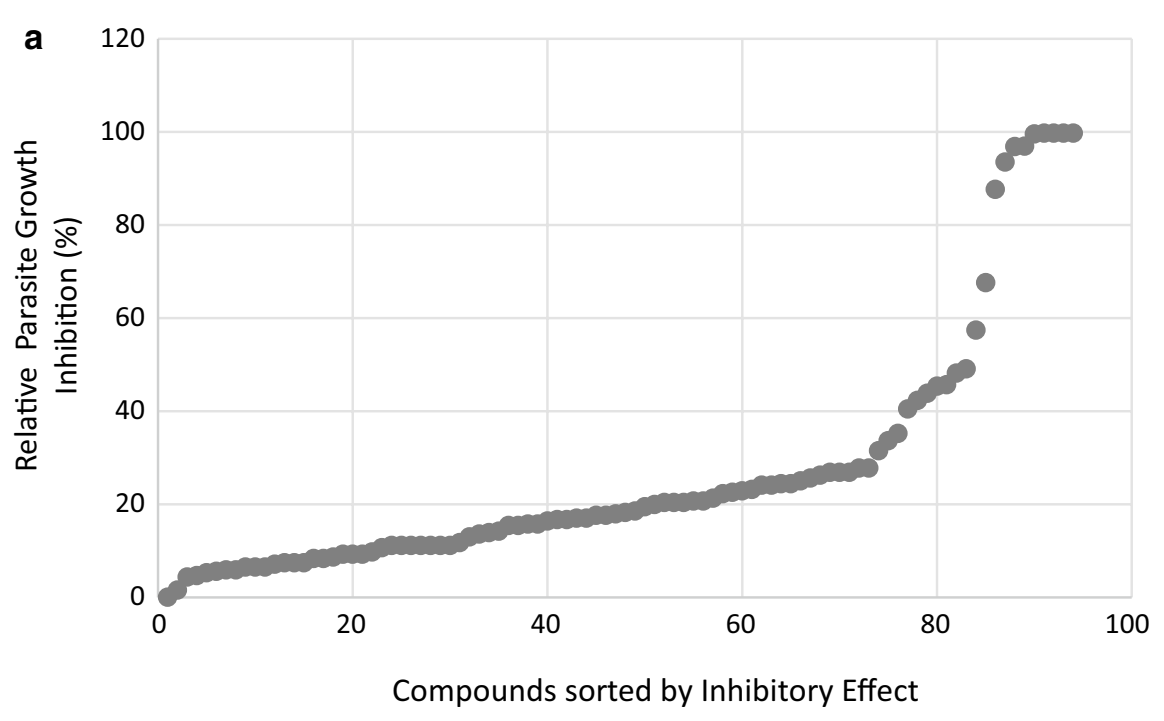

b

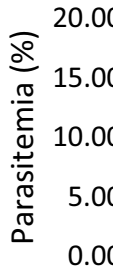

Berberine chloride

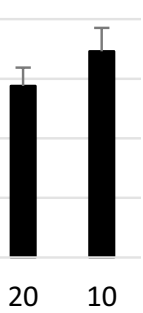

$\begin{array}{lll}10000 & 80 & 60\end{array}$

Palmatine chloride

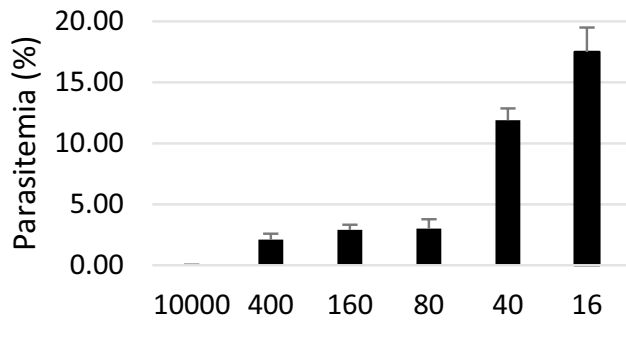

Coptisine chloride

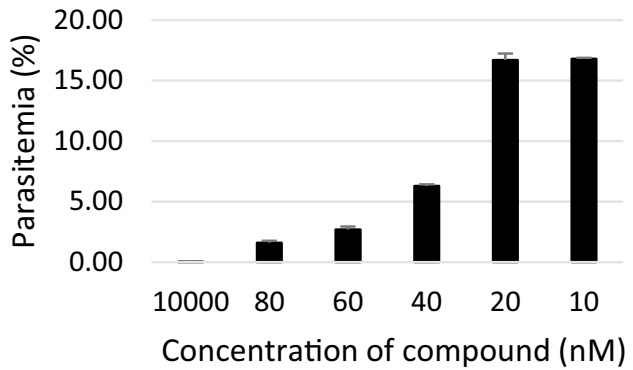

Dehydrocorydaline nitrate

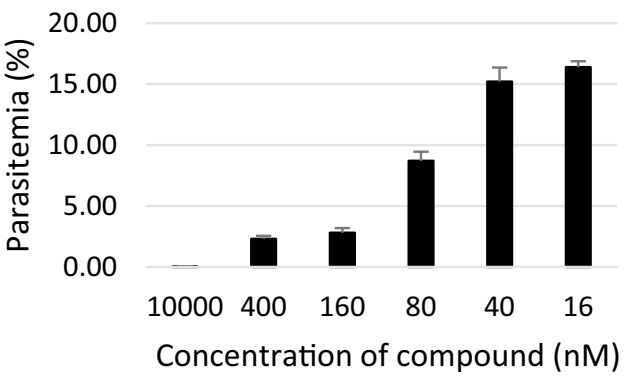

Fig. 1 Natural compound screening for anti-malarial activity using 3D7 strain. a Plasmodium falciparum 3D7 strain-infected human RBCs were incubated with $10 \mu \mathrm{M}$ test compounds (96 different compounds) for $96 \mathrm{~h}$ (see "Methods"). Five compounds showed almost 100\% growth inhibitory effects. $\mathbf{b}$ The four selected compounds (berberine chloride, coptisine chloride, palmatine chloride, and dehydrocorydaline nitrate) were investigated for concentration-dependent growth inhibition effects. Six concentrations of each compound were incubated with parasites for $96 \mathrm{~h}$. Concentration-dependent anti-malarial effects were observed and $I C_{50}$ values were calculated (see Table 1) 
Table 1 Hit compounds from the first screening using $P$. falciparum 3D7 strain

\begin{tabular}{ll}
\hline Compound & $\begin{array}{l}\text { Growth inhibition rate (\%) } \\
\text { IC }_{\mathbf{5 0}} \text { (SEM) (nM) }\end{array}$ \\
\hline Palmatine chloride & 99.5 \\
& $26(24-28)$ \\
Berberine chloride & 99.7 \\
& $36(34-38)$ \\
Coptisine chloride & 99.7 \\
& $35(34-36)$ \\
Dehydrocorydaline nitrate & 99.7 \\
Timosaponin A-III & $38(36-39)$ \\
& 99.7 \\
& Not determined due to its \\
& haemolytic effect \\
\hline
\end{tabular}

b). In addition, the authors calculated the $P$. falciparum selectivity of these compounds and extracts, and found that all four compounds had $>100$ selectivity and that the two extracts had $\leq 10$ selectivity (Table 5 ).

The results suggest that these compounds and extracts may be safe as anti-malarial drugs because they showed strong effects against only parasites and had little effect on human cells.

\section{Stage-specific growth inhibition}

Stage-specific growth inhibition activities of the compounds and extracts were also investigated using $P$. falciparum 3D7 strain. Berberine chloride, dehydrocorydaline nitrate, and palmatine chloride showed growth inhibition effects during the trophozoite and schizont stages (Fig. 6a). Berberine chloride showed a particularly strong effect during the trophozoite stage. Coptisine chloride showed an effect during the trophozoite stage, but may also exhibit an effect during the schizont stage (Fig. 6a). Phellodendri cortex and Coptidis rhizoma also showed effects during the trophozoite and schizont stages (Fig. 6b), and their effects were stronger than those of the natural compounds. The authors observed growth inhibitory effects during the trophozoite and schizont stages with both the compounds and extracts.

\section{Discussion}

Here the authors identified compounds and extracts with anti-malarial activity from a library of TCMs. Antimalarial activity for berberine has been reported previously, but its intensity differed from that obtained in this study. In the authors' investigation, the $\mathrm{IC}_{50}$ of berberine chloride was $31 \mathrm{nM}$; however, other studies reported $\mathrm{IC}_{50}$

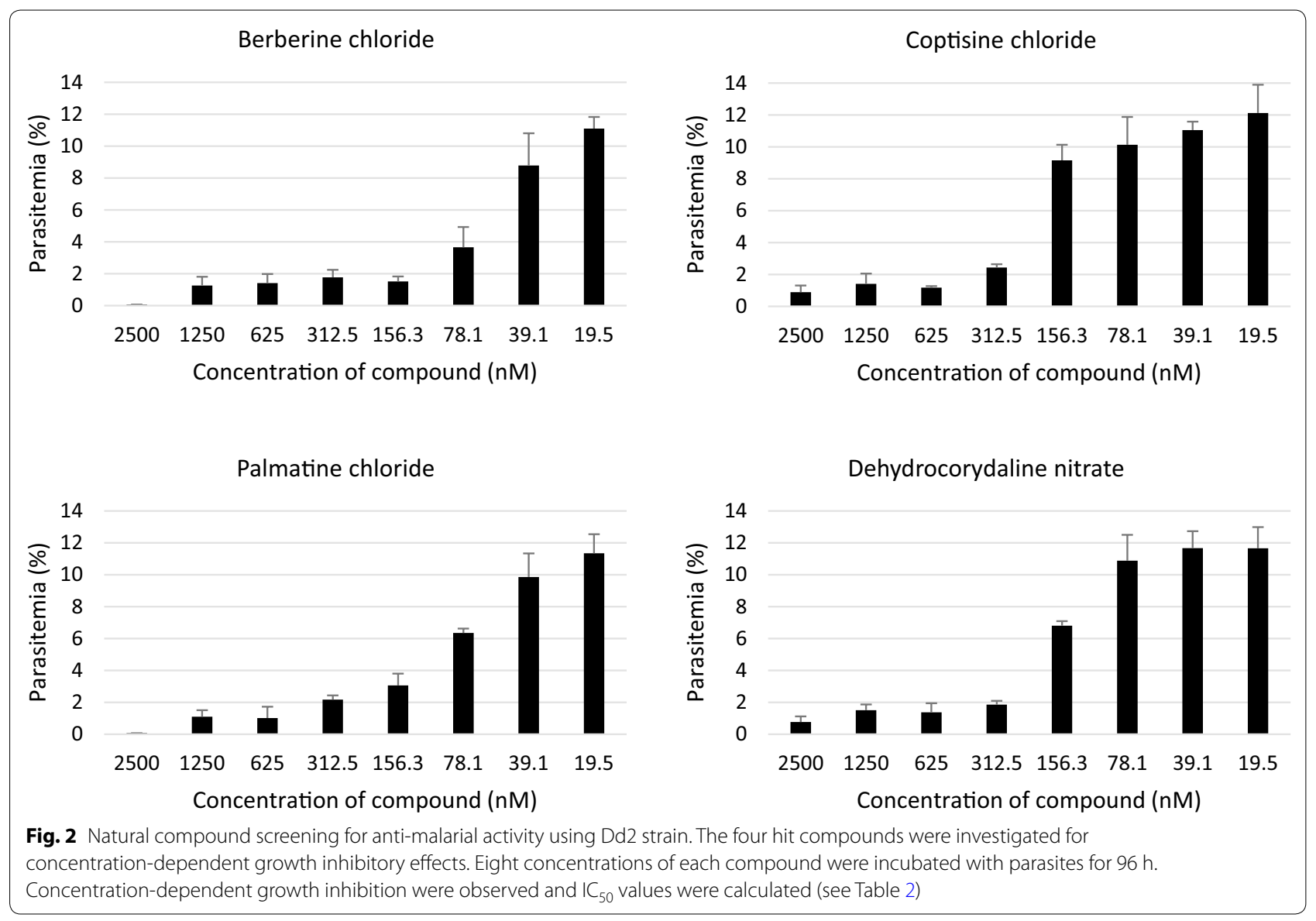


Table 2 Hit compounds screening using P. falciparum Dd2 strain

\begin{tabular}{|c|c|}
\hline Compound & $\begin{array}{l}\text { Growth } \\
\text { inhibitory } \\
\text { rate (\%) } \\
\mathrm{IC}_{50}(\mathrm{SEM}) \\
(\mu \mathrm{g} / \mathrm{ml})\end{array}$ \\
\hline Berberine chloride & $\begin{array}{l}100 \\
54(50-59)\end{array}$ \\
\hline Palmatine chloride & $\begin{array}{l}100 \\
81(75-87)\end{array}$ \\
\hline Dehydrocorydaline nitrate & $\begin{array}{l}94.5 \\
161(149-174)\end{array}$ \\
\hline Coptisine chloride & $\begin{array}{l}93.5 \\
190(172-210)\end{array}$ \\
\hline
\end{tabular}

values of $270 \mathrm{nM}$ (using FCR3 strain, cultured for $72 \mathrm{~h}$ ) [12], <100 nM (using 3D7 strain, cultured for $72 \mathrm{~h}$ ) [13], $80 \mathrm{nM}$ (using K39 strain, cultured for $66 \mathrm{~h}$ ), and $1.98 \mu \mathrm{M}$ (using V1/S strain, cultured for $66 \mathrm{~h}$ ) [14]. In these studies, different strains of $P$. falciparum were used and the culture time was also shorter than the authors.' The FCR3 and V1/S strains have multi-drug resistance (FCR3 is resistant to CQ and cycloguanil; V1/S is resistant to CQ, $\mathrm{SD}$, and PYR). In contrast, the 3D7 and K39 strains are not multi-drug resistant (3D7 is resistant to SD; K39 is resistant to PYR). Growth of Dd2 strain (resistance to CQ and mefloquine) was inhibited at a little higher drug concentration, suggesting that difference in parasite strains did not much contribute the difference in $\mathrm{IC}_{50}$ values. With respect to culture time, the authors' experiments

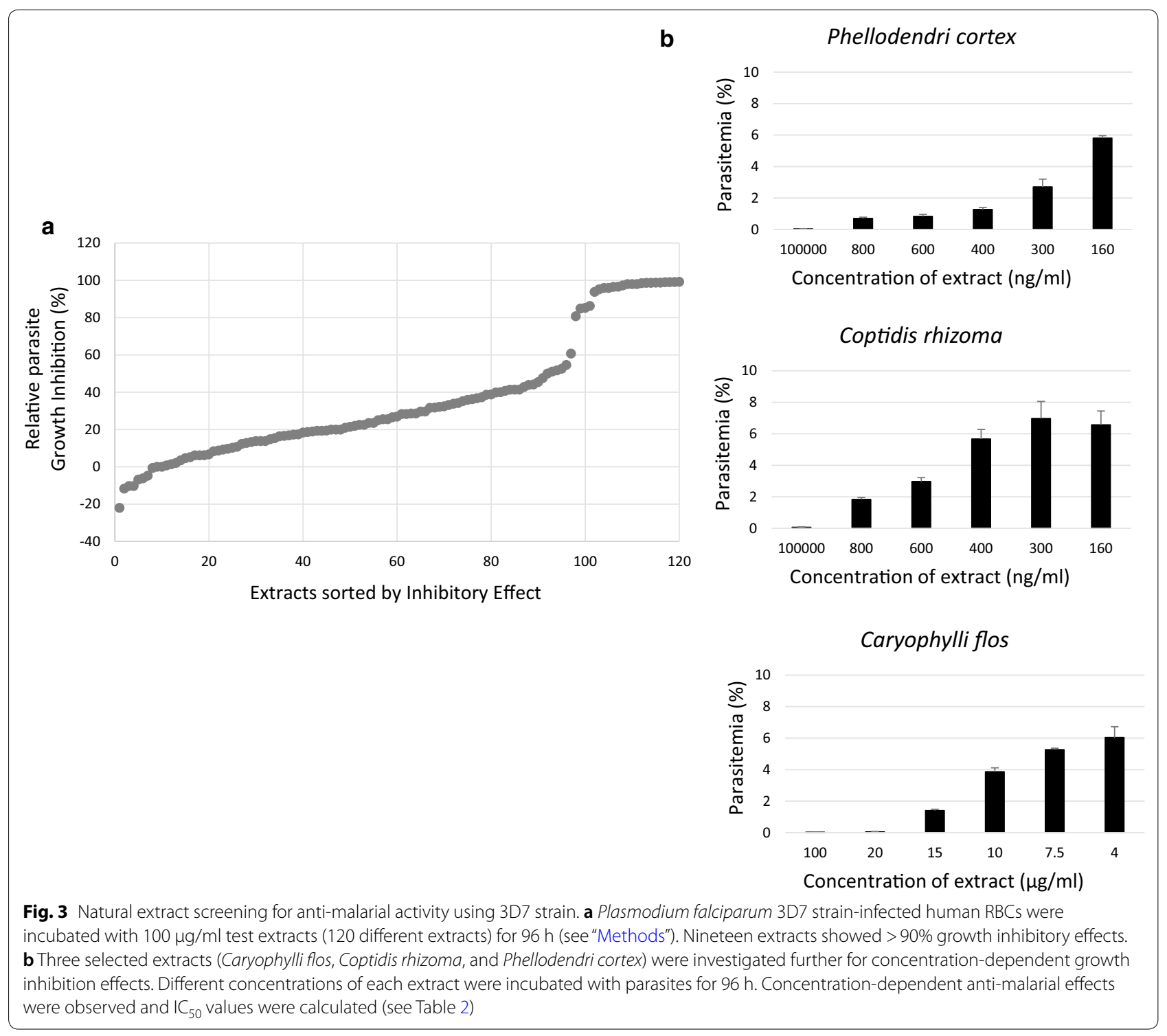


Table 3 Hit extracts from the first screening using $P$. falciparum 3D7 strain

\begin{tabular}{ll}
\hline Extract & $\begin{array}{l}\text { Growth inhibition rate (\%) } \\
\text { IC }_{\mathbf{5 0}}(\mathbf{S E M})(\boldsymbol{\mu g} / \mathbf{m l})\end{array}$ \\
\hline Forsythiae fructus & 98.6 \\
& $>20$ \\
Myrrha & 98.8 \\
Rhei rhizoma & $>20$ \\
& 98.8 \\
Quercus cortex & $>20$ \\
& 99.0 \\
Spatholobicaulis & $>20$ \\
Caryophylliflos & 99.0 \\
& $>20$ \\
Coptidis rhizoma & 98.6 \\
& $11.5(11.1-11.8)$ \\
Phellodendri cortex & 99.1 \\
& $633 \times 10^{-3}(601-665)$ \\
& 99.2 \\
\hline
\end{tabular}

were performed for $96 \mathrm{~h}$, whereas the others were performed within $72 \mathrm{~h}$. This means that the authors' parasites were exposed to the natural products twice, because the life cycle of malaria is $48 \mathrm{~h}$, but only once in these other studies. Therefore, the authors believe that these differences in $\mathrm{IC}_{50}$ values resulted from the differences in the culture times used.

The authors' study showed that berberine chloride, coptisine chloride, palmatine chloride, and dehydrocorydaline nitrate have strong anti-malarial activity; their base compounds (berberine, coptisine, palmatine, and dehydrocorydaline) belong to the isoquinoline alkaloids. Berberine and its analogues, which include dehydrocorydaline, are called protoberberine alkaloids and their anti-malarial activity has been reported previously [11, 12, 15-17]. However, anti-malarial activity of dehydrocorydaline nitrate has not been previously demonstrated even though it is a protoberberine alkaloid. The authors' study is thus the first to reveal this activity of dehydrocorydaline nitrate. This result also highlights the possibility for discovery of other as yet unrevealed natural products that could contribute to overcoming malaria disease. Because the molecular structures of coptisine and palmatine are similar to that of berberine, these two compounds can be regarded as members of the protoberberine alkaloids. Thus, protoberberine alkaloids may have any common sites or moiety that performs the antimalarial effect observed.

Few studies have analysed natural extracts for antimalarial activity. Phellodendri cortex, Coptidis rhizoma, and Caryophylli flos have not been directly evaluated in such studies even though they have been used as a source of extracts such as berberine. The authors' study is the first to investigate the anti-malarial activity of the three extracts, and the authors discovered that they do indeed have growth inhibitory effects on malaria parasites. In particular, Phellodendri cortex has a low $\mathrm{IC}_{50}$ value and high selectivity, suggesting it may be of value in developing a novel strategy against malaria. Indeed, Phellodendri cortex includes berberine and palmatine, and Coptidis rhizoma includes berberine, palmatine, and coptisine. Therefore, the activity of these two natural

Table 4 Hit extracts screening using $P$. falciparum Dd2 strain

Extract $\quad$ Growth inhibition rate (\%) $\mathrm{IC}_{50}(\mathrm{SEM})(\mu \mathrm{g} / \mathrm{ml})$

Coptidis rhizoma 100

$827 \times 10^{-3}(463-1474)$

Phellodendricortex $\quad 98.8$

$651 \times 10^{-3}(384-1107)$
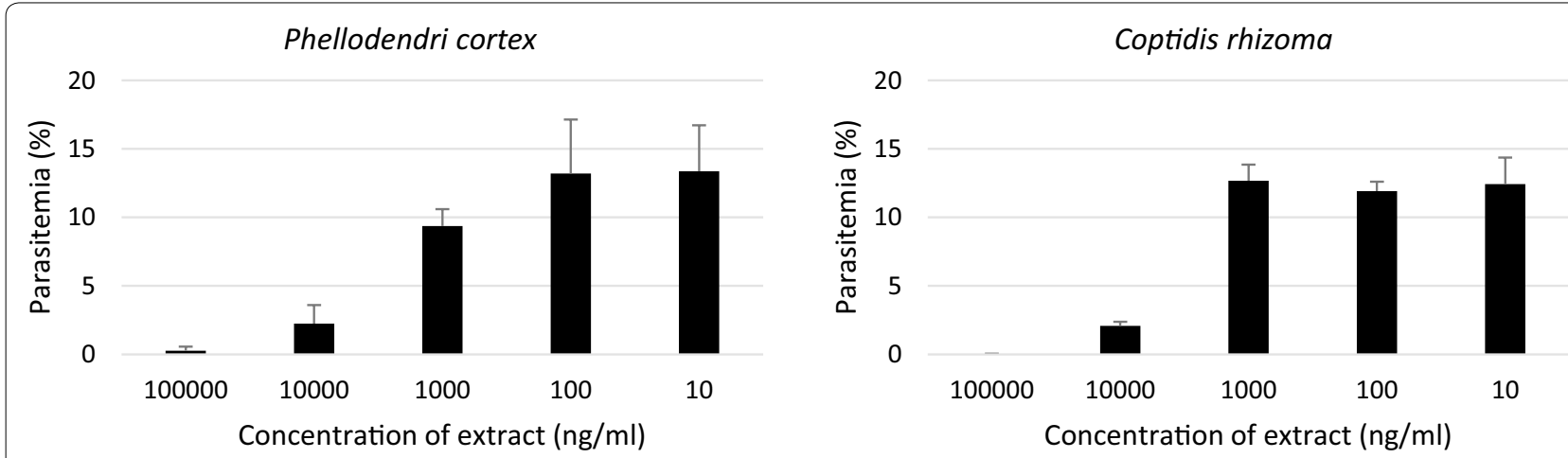

Fig. 4 Natural extract screening for anti-malarial activity using Dd2 strain. The two hit extracts were investigated for concentration-dependent growth inhibitory effects. Five concentrations of each extract were incubated with parasites for $96 \mathrm{~h}$. Concentration-dependent growth inhibition were observed and $I C_{50}$ values were calculated (see Table 4) 
a

Compound cytotoxicity assay

120

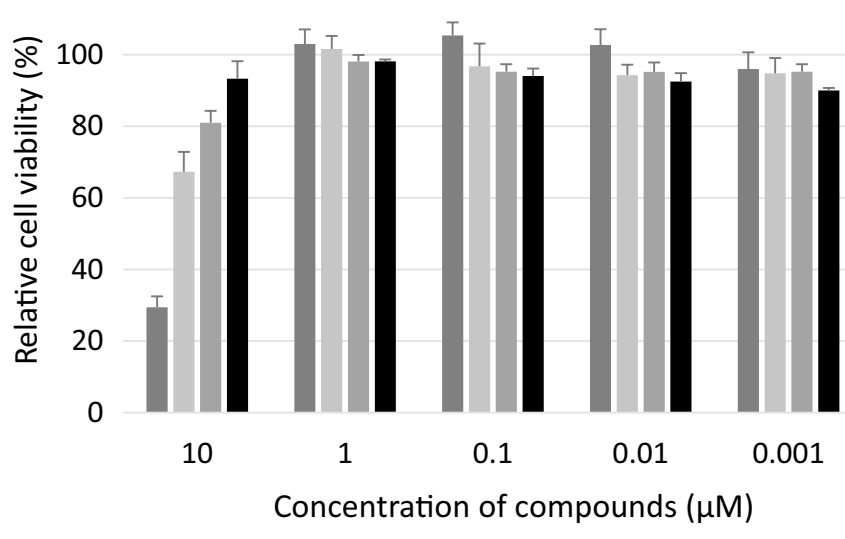

b

Extracts cytotoxicity assay 120

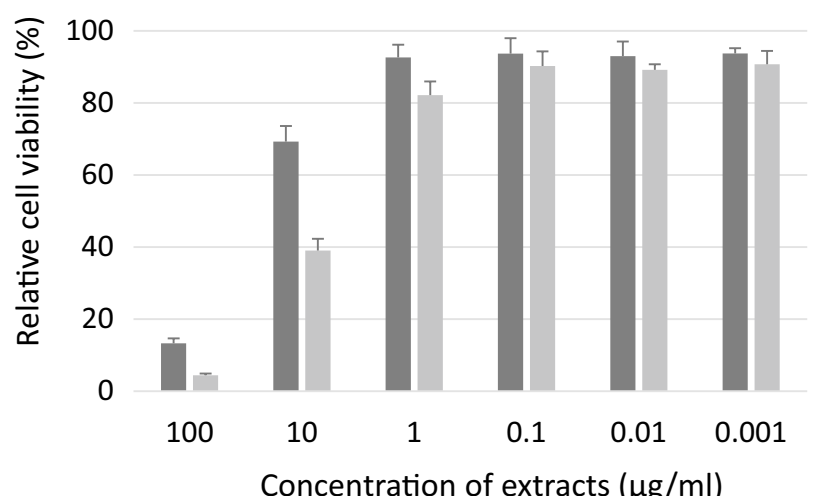

- Phellodendri cortex Coptidis rhizoma

Fig. 5 Cytotoxicity of the natural compounds and extracts. a Cytotoxicity assays were performed with the four anti-malarial compounds. 293T cells were incubated with different concentrations of test compounds for $96 \mathrm{~h}$ (see "Methods"). The authors observed high cell viability in the presence of all four compounds at concentrations of $<1 \mu \mathrm{M}$. b Cytotoxicity assays were performed with the two anti-malarial extracts. $293 \mathrm{~T}$ cells were incubated with various concentrations of the test extracts for $96 \mathrm{~h}$ (see "Methods"). The authors observed high cell viability in the presence of both extracts at concentrations of $<1 \mu \mathrm{g} / \mathrm{ml}$

Table 5 Parasite selectivity of hit compounds and extracts

\begin{tabular}{lc}
\hline Compounds and extracts & Selectivity (\%) \\
\hline Palmatine chloride & $>45,500$ \\
Coptisine chloride & $>29,400$ \\
Berberine chloride & 24,200 \\
Dehydrocorydaline nitrate & $>14,900$ \\
Phellodendri cortex & 11,000 \\
Coptidis rhizoma & 1000 \\
Caryophylliflos & 300 \\
\hline
\end{tabular}

extracts may be caused by the isoquinoline alkaloids they contain. In contrast, the other six extracts of the eight the authors studied in detail do not include isoquinoline alkaloids. Moreover, one component of Quercus cortex is an uncharacterized entity. Therefore, the authors may discover additional novel anti-malarial compounds from TCMs in the future.

The authors cultured P. falciparum 3D7 strain parasites in culture medium with 10 times the $\mathrm{IC}_{50}$ value of berberine chloride for 3 months in an attempt to produce a berberine-resistant malaria parasite, but the authors 


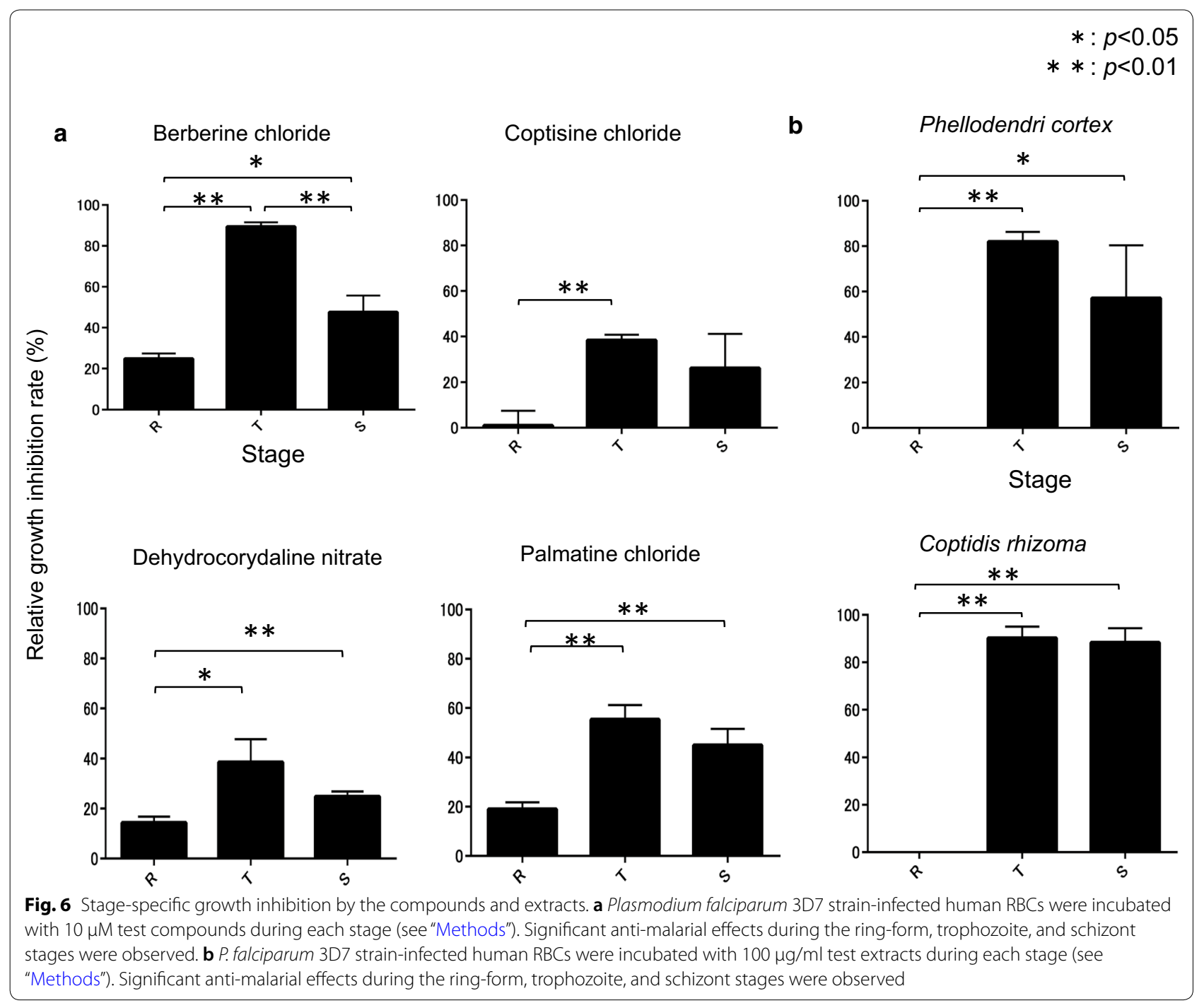

were unable to obtain such a parasite. A previous study reported that berberine inhibits the telomerase activity of P. falciparum K1 strain [18, 19]. Telomeres play important roles in eukaryotic cells in maintaining genome stability and ensuring accurate DNA replication. It may be difficult to maintain cells without these functions, so the fact that the authors were unable to create the resistant parasite suggests that berberine and other analogues may be instrumental in the development of novel antimalarial drugs because these compounds inhibit the telomerase activity of $P$. falciparum but not host cells at low concentration (Table 5).

Extracts showed stronger anti-malarial effect than compounds during trophozoite and schizont stages. This difference may be caused by the difference between a single compound and a complex extract, in other words, the power of the extract complex was stronger than that of the individual compounds. Few studies have evaluated the stage-specific anti-malarial effects of natural products; therefore, the authors' study may help elucidate the mechanism of the inhibitory effect. The authors observed growth inhibitory effects during the trophozoite and schizont stages with both the compounds and extracts. The authors' result suggests that these natural products use an inhibitory mechanism that suppresses a development process of the plasmodium parasite, such as gene expression or DNA replication.

\section{Conclusions}

The authors identified four compounds (berberine chloride, coptisine chloride, palmatine chloride, and dehydrocorydaline nitrate) and two extracts (Phellodendri cortex and Coptidis rhizoma) that show anti-malarial activity by screening a library of TCMs. The authors' study is the 
first to determine that dehydrocorydaline nitrate, Phellodendri cortex, and Coptidis rhizoma can inhibit the growth of both 3D7 and Dd2 strains of malaria parasites. The $\mathrm{IC}_{50}$ values obtained for these compounds were equivalent to that of CQ [9] and better than that of PYR [10]. The authors' findings indicate that these compounds and extracts derived from TCMs may be of value in the development of new anti-malarial drugs.

\section{Abbreviations}

ACT: artemisinin-based combination therapy; TCM: traditional Chinese medicine; $\mathrm{RBC}$ : red blood cell; $\mathrm{GIA}$ : growth inhibition assay; $I_{50}$ : half maximum inhibitory concentration; ART: artemisinin; ATN: artesunate; QN: quinine; QND: quinidine; CQ: chloroquine; SD: sulfadoxine; PYR: pyrimethamine.

\section{Authors' contributions}

KK designed the study. MN, YM, YH, MHBK and RT did laboratory work and analysed the data. MN was a major contributor in writing the manuscript. All authors read and approved the final manuscript.

\section{Acknowledgements}

The authors thank the Institute of Natural Medicine (The University of Toyama, Toyama, Japan) for providing the natural drug library.

\section{Competing interests}

The authors declare that they have no competing interests.

\section{Availability of data and materials}

The datasets used or analysed during the current study are available from the corresponding author on reasonable request.

\section{Consent for publication}

Not applicable.

\section{Ethics approval and consent to participate} Not applicable.

\section{Funding}

This research was supported by a Grant-in-Aid for the Cooperative Research Project from Institute of Natural Medicine, University of Toyama in 2017; by a JSPS Research Fellowship for Young Scientists, Grant-in-Aid for Scientific Research from the Ministry of Education, Culture, Science, Sports, and Technology (MEXT) of Japan; by the Program to Disseminate Tenure Tracking System and the Adaptable \& Seamless Technology Transfer Program through Targetdriven R\&D (A-STEP) from the Japan Science and Technology Agency (JST); and by the Suhara Kinen Zaidan and Takeda Science Foundation.

\section{Publisher's Note}

Springer Nature remains neutral with regard to jurisdictional claims in published maps and institutional affiliations.

Received: 29 November 2017 Accepted: 15 June 2018

Published online: 25 June 2018

\section{References}

1. WHO. World malaria report 2016. Geneva: World Health Organization; 2016.

2. Singh B, Kim Sung L, Matusop A, Radhakrishnan A, Shamsul SSG, CoxSingh J, et al. A large focus of naturally acquired Plasmodium knowlesi infections in human beings. Lancet. 2004;363:1017-24.

3. Miller $L H$, Su X. Artemisinin: discovery from the Chinese herbal garden. Cell. 2011;146:855-8.
4. Menard D, Dondorp A. Antimalarial drug resistance: a threat to malaria elimination. Cold Spring Harb Perspect Med. 2017;7:025619.

5. Schlitzer M. Malaria chemotherapeutics part I: history of antimalarial drug development, currently used therapeutics, and drugs in clinical development. ChemMedChem. 2007;2:944-86.

6. Cheng JT. Drug therapy in Chinese traditional medicine. J Clin Pharmacol. 2000;40:445-50.

7. Recuenco FC, Kobayashi K, Ishiwa A, Enomoto-Rogers Y, Fundador NGV, Sugi T, et al. Gellan sulfate inhibits Plasmodium falciparum growth and invasion of red blood cells in vitro. Sci Rep. 2014;4:4723.

8. Skinner TS, Manning LS, Johnston WA, Davis TM. In vitro stage-specific sensitivity of Plasmodium falciparum to quinine and artemisinin drugs. Int J Parasitol. 1996;26:519-25.

9. Kiara SM, Okombo J, Masseno V, Mwai L, Ochola I, Borrmann S, et al. In vitro activity of antifolate and polymorphism in dihydrofolate reductase of Plasmodium falciparum isolates from the Kenyan Coast: emergence of parasites with lle-164-Leu mutation. Antimicrob Agents Chemother. 2009;53:3793-8.

10. Okombo J, Kiara SM, Rono J, Mwai L, Pole L, Ohuma E, et al. In vitro activities of quinine and other antimalarials and pfnhe polymorphisms in Plasmodium isolates from Kenya. Antimicrob Agents Chemother. 2010;54:3302-7.

11. Iwasa K, Kim HS, Wataya Y, Lee DU. Antimalarial activity and structureactivity relationships of protoberberine alkaloids. Eur J Med Chem. 1998:33:65-9

12. Iwasa K, Nishiyama Y, Ichimaru M, Moriyasu M, Kim H-S, Wataya Y, et al. Structure-activity relationships of quaternary protoberberine alkaloids having an antimalarial activity. Eur J Med Chem. 1999;34:1077-83.

13. Hayase H, Watanabe N, Lim CL, Nogawa T, Komatsuya K, Kita K, et al. Inhibition of malaria parasite growth by quinomycin A and its derivatives through DNA-intercalating activity. Biosci Biotechnol Biochem. 2015;79:633-5.

14. Silikas N, Mccall DLC, Sharples D, Watkins WM, Waigh RD, Barber J. The antimalarial activity of berberine and some synthetic analogues. Pharm Pharmacol Commun. 1996;2:55-8.

15. Wright CW, Marshall SJ, Russell PF, Anderson MM, Phillipson JD, Kirby GC, et al. In vitro antiplasmodial, antiamoebic, and cytotoxic activities of some monomeric isoquinoline alkaloids. J Nat Prod. 2000;63:1638-40.

16. Grycová L, Dostál J, Marek R. Quaternary protoberberine alkaloids. Phytochemistry. 2007:68:150-75.

17. Marek R, Seckárová P, Hulová D, Marek J, Dostál J, Sklenár V. Palmatine and berberine isolation artifacts. J Nat Prod. 2003;66:481-6.

18. Parida P, Kalita S, Yadav RNS, Shankar B. Homology modeling and docking studies of Plasmodium falciparum telomerase reverse transcriptase with berberine and some of its derivatives. Bangladesh J Pharmacol. 2014;9:96-104.

19. Sriwilaijareon N, Petmitr S, Mutirangura A, Ponglikitmongkol M, Wilairat P. Stage specificity of Plasmodium falciparum telomerase and its inhibition by berberine. Parasitol Int. 2002;51:99-103.

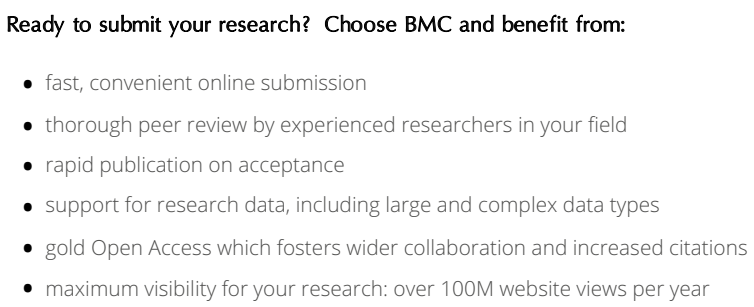

